\title{
No evidence on the effectiveness of oral splints for the management of temporomandibular joint dysfunction pain in both short and long-term follow-up systematic reviews and meta-analysis studies
}

\author{
Atef Abdel Hameed Fouda \\ Department of Maxillofacial Surgery, Kasr El Eyne Hospital, Cairo University, Cairo, Egypt
}

\begin{abstract}
J Korean Assoc Oral Maxillofac Surg 2020;46:87-98)
The aim of this study was to determine the efficacy of oral splints in reducing the intensity of pain in patients with temporomandibular joint dysfunction in both short and long-term treatment durations. Electronic databases, Cochrane Library, MEDLINE via PubMed, Web of Science, Egyptian Knowledge Bank, and EMBASE were searched for randomized controlled trials comparing different types of splints to non-occluding splints, behavioral therapy, pharmacotherapy, counseling, and no treatment. The risk of bias was assessed by using Cochrane risk of bias recommendations. Fixed and random effects were used to summarize the outcomes. The effect estimates were expressed as standardized mean differences (SMD) or risk ratios with a $95 \%$ confidence interval (CI). Subgroup analyses were carried out according to the treatment duration. Twenty-two studies met the inclusion criteria. A meta-analysis of short-term studies up to three months revealed no significant difference between the study groups. However, long-term studies exhibited a significant difference in pain reduction in favor of the control group. Total analysis revealed that the control group resulted in significant pain reduction ( $\mathrm{SMD} 0.14,95 \% \mathrm{CI} 0.05-0.23, P=0.002, \mathrm{I}^{2}=0 \%$ ). Oral splints are not effective in reducing pain intensity or improving function in patients with temporomandibular joint dysfunction.
\end{abstract}

Key words: Occlusal splints, Pain, Review, Meta-analysis

[paper submitted 2019. 9. 20 / revised 2019. 11. 30 / accepted 2019. 12. 17]

\section{Introduction}

Psychologic, social, and biologic factors are several causes of temporomandibular joint dysfunction (TMJD). The relief of pain and the ability to masticate are considered the principal concerns for patients. An understanding of the psychological conditions with proper treatments for chronic pain and dysfunction must be considered ${ }^{1}$. The ultimate success was achieved when the operator met the patients' expectations by improving their quality of life ${ }^{2}$.

Successful treatment of TMJD depends on an accurate diagnosis followed by the selection of proper treatment. Treat-

\footnotetext{
Atef Abdel Hameed Fouda

Department of Maxillofacial Surgery, Kasr El Eyne Hospital, Cairo University, Manial, Cairo 12111, Egypt

TEL: +20-1093440600 FAX: +20-23642938

E-mail: Atef.fouda@dentistry.cu.edu.eg

ORCID: https://orcid.org/0000-0001-6386-3696

(c) This is an open-access article distributed under the terms of the Creative Commons Attribution Non-Commercial License (http://creativecommons.org/ licenses/by-nc/4.0/), which permits unrestricted non-commercial use, distribution, and reproduction in any medium, provided the original work is properly cited. Copyright (C) 2020 The Korean Association of Oral and Maxillofacial Surgeons. All rights reserved.
}

ment modalities applied to relieve pain and other symptoms of this disorder are classified into non-invasive physical therapy $^{3}$, pharmacotherapy ${ }^{4}$, counseling assurance ${ }^{5}$, and occlusal splint therapy ${ }^{6}$. Minimally invasive arthrocentesis ${ }^{7}$, acupuncture $^{8}$, and lasers ${ }^{9}$ are among the invasive surgical intervention methods ${ }^{10}$.

Occlusal splints are considered a simple, chair-side, and less invasive treatment. Occlusal splints are the favorable treatment of choice in our institute after medications. The clinical findings of occlusal splints for the treatment of both myofascial pain dysfunction (MPD) syndrome and disk displacement are controversial and they may act as a placebo rather than a specific therapeutic mechanism ${ }^{11}$.

Regardless of the splint mode of action, many old and several recent clinical trials and systematic reviews have documented its therapeutic effectiveness in reducing pain intensity and improving masticatory function in patients with painful temporomandibular disorder (TMD) ${ }^{12,13}$. However, most of the current literature did not demonstrate any beneficial effect in short-term ${ }^{14}$ and long-term follow-up ${ }^{15}$.

Among the causes of variability between the results, pa- 
tients with TMJD cannot be considered a homogeneous group and will respond differently, with investigators relying on subjective symptoms reported by the patients. Moreover, studies can present with inadequate sample sizes, short follow-up periods, poor quality of the control group, and bias in data reporting. Proper evaluation of splint appliance therapy is achieved by applying long-term follow-ups, randomized controlled study designs, recruitment of a homogeneous population, and blinding in data extraction.

The investment in evidence-based medicine and its role in improving the acceptance of studies for publication, besides the natural progress of the disease, recent diagnostic measures, and improvement of communication between clinicians and patients means it is likely these account for changes in the old concepts that previously guided temporomandibular joint $(\mathrm{TMJ})$ treatment.

This review was conducted to shed light on doubts about the therapeutic value of oral splints through a meta-analysis of data from different relevant randomized controlled trials (RCTs) and studies of different types of occlusal splints. Our purpose was to evaluate the effectiveness of splint therapy in ameliorating pain issues in patients with TMJD.

\section{Materials and Methods}

The recommendations from the PRISMA (Preferred Reporting Items for Systematic Reviews and Meta-Analyses) statement were followed ${ }^{16}$.

\section{Eligibility criteria}

1) Inclusion criteria

RCT studies that were conducted in humans over the past twenty years. Patients with TMJD that included myofascial pain with the source of pain being either muscular or from the joint, and patients with disk displacements with or without reduction. Studies that compared splints to non-invasive treatment (medications-biofeedback, counseling, and nonoccluding splints). Patient diagnosis was either based on Research Diagnostic Criteria for Temporomandibular Disorders (RDC/TMD) or clinical examination.

\section{2) Excluded from the study}

Published studies older than twenty years, studies on animals, uncontrolled studies comparing different types of splints with each other, or studies with minimally invasive treatment modalities (low-level laser, arthrocentesis, acu- puncture, and physiotherapy). If there were multiple comparisons within the same study, each comparison was included separately. Moreover, various intervals within the related research were recorded in each relevant subgroup.

\section{Population}

Patients with myofascial pain and/or disk displacement with or without reduction were included. Patients with arthritis or neuralgias were excluded.

\section{Intervention}

The study included all types of splints (stabilizing splint, Michigan splint, centric relation appliance, flat occlusal appliance, soft or hard splints, vinyl appliances, and positioning splints).

\section{Control group}

The patients in the control group underwent medical treatment, biofeedback, non-occluding splints, massages, behavioral therapy, cognitive, counseling, or no treatment.

\section{Outcome}

1) Primary outcome

Pain intensity was estimated with any recognized, validated pain scale: visual analogue scale, numeric rating scale, characteristic pain intensity, and symptom severity index. Subgroup analyses were carried out for the outcomes based on the follow-up duration classified into one, two, three, six, and twelve months.

2) Secondary outcome

Maximum mouth opening (MMO) was evaluated by interincisal opening measured in millimeters.

\section{Search methods for studies identification}

The search for studies in the English language was conducted up to August 2018. The following databases were searched individually from 1998 to the present: MEDLINE, Web of Science, EMBASE, and Egyptian Knowledge Bank. Manual searches, references from primary studies, and systematic reviews for relevant data were obtained. The search used a combination of controlled vocabulary: 'temporomandibular 
joint disorders'[MeSH Terms] OR 'temporomandibular'[All Fields], AND 'joint'[All Fields], AND 'disorders'[All Fields], OR 'temporomandibular joint disorders'[All Fields], OR ('temporomandibular'[All Fields] AND 'disorders'[All Fields]) OR 'temporomandibular disorders'[All Fields], OR 'mouth'[MeSH Terms] OR 'mouth'[All Fields] OR 'oral'[All Fields]) AND ('splints'[MeSH Terms] OR 'splints'[All Fields] OR 'splint'[All Fields]). 'temporomandibular joint'[MeSH Terms] OR ('temporomandibular'[All Fields] AND 'joint'[All Fields]) OR 'temporomandibular joint'[All Fields] OR 'TMJ'[All Fields], AND 'pain'[MeSH Terms] OR 'pain'[All Fields].

\section{Data extraction and management}

Both continuous (mean, standard deviation) and dichotomous (event, control) studies were included in the analysis.

\section{Assessment of study risk of bias}

All included studies were evaluated with consent from the Cochrane Handbook for Systematic Reviews of Interventions version 5.1.0 ${ }^{17}$. The risk of bias was assessed using Cochrane Collaboration's tool with response options of 'low risk', 'unclear risk', and 'high risk' for the following criteria: sequence generation, allocation concealment, blinding, incomplete outcome data, reporting bias, and other biases.

\section{Measurements of the treatment effect}

\section{1) Pain intensity}

Standardized mean difference (SMD) with 95\% confidence interval (CI) and fixed effects were used for continuous outcomes. For dichotomous outcomes, the estimate of the effect was expressed as a risk ratio (RR) together with $95 \%$ CIs and random effects.

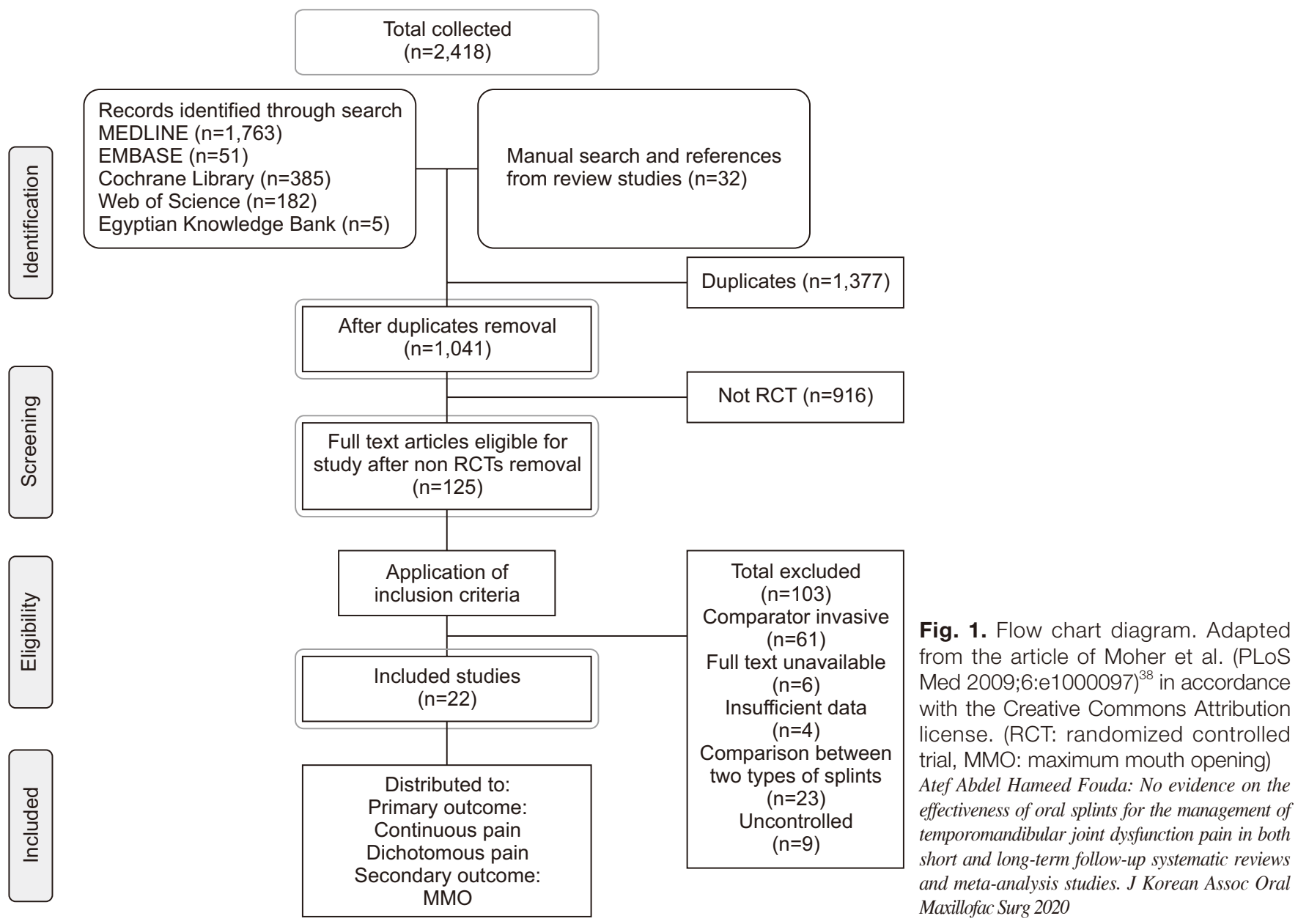


2) Maximum mouth opening

The mean difference (MD) with 95\% CI and fixed effects was used for the assessment of the outcomes.

\section{3) Assessment of heterogeneity}

Chi-square testing for heterogeneity was performed, and the extent of the inconsistency of the treatment effects $\left(\mathrm{I}^{2}\right)$ across the trials was measured. We considered heterogeneity substantial if $\mathrm{I}^{2}$ was greater than $30 \%$, or if there was a low $P$ value (less than 0.10 ) in the chi-square test for heterogeneity. We used a fixed-effect meta-analysis for combining the data, but clinical heterogeneity random-effect was used in the subgroup analyses.

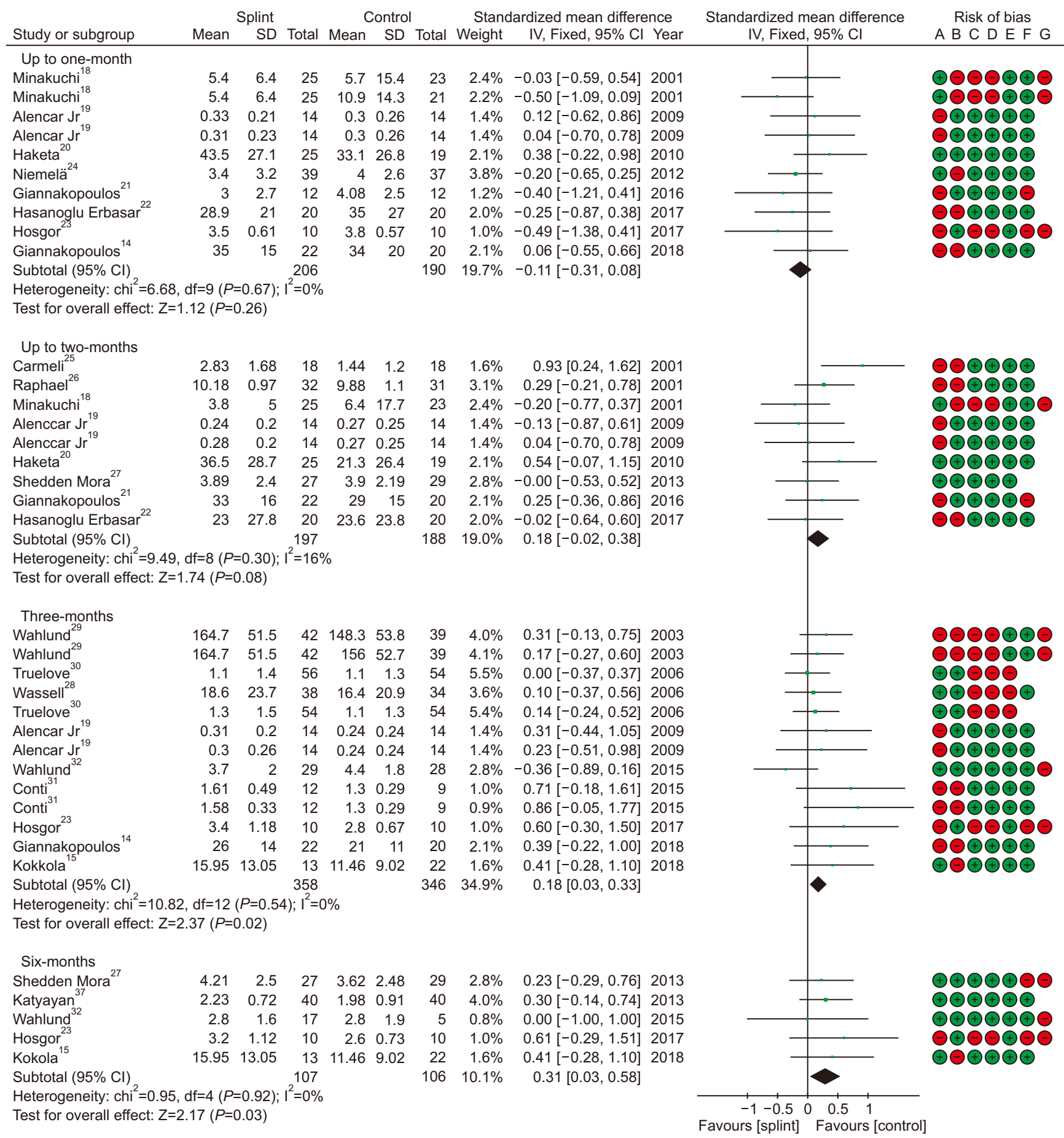

Fig. 2. Forest plot of continuous pain analysis at different intervals with risk of bias for the included studies. (SD: standard deviation, Cl: confidence interval, df: degree of freedom) 


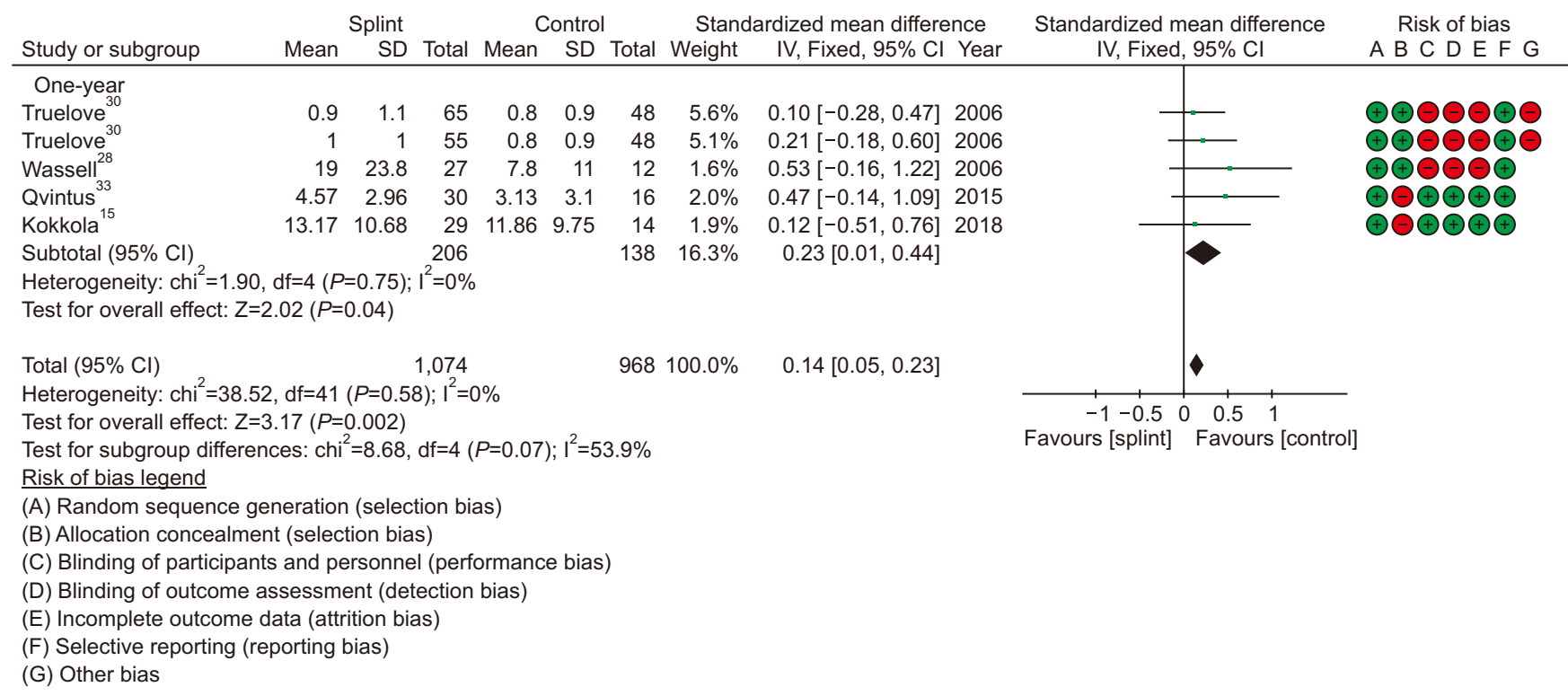

Fig. 2. Continued.

Atef Abdel Hameed Fouda: No evidence on the effectiveness of oral splints for the management of temporomandibular joint dysfunction pain in both short and long-term follow-up systematic reviews and meta-analysis studies. J Korean Assoc Oral Maxillofac Surg 2020

\section{4) Assessment of reporting biases}

Publication bias was appraised using the symmetry of the funnel plots. We visually assessed funnel plot asymmetry. When asymmetry was detected, investigations were performed using exploratory analyses.

\section{5) Methodological quality assessment}

Data synthesis and meta-analyses were performed according to the rules adopted by the Cochrane Collaboration reviewer's handbook ${ }^{17}$. All statistical analyses were conducted using Review Manager software (RevMan 5.3).

\section{Results}

The initial search yielded a total of 2,418 titles: 1,763 from PubMed, 51 from EMBASE, 385 from the Cochrane Central library, 182 from the Web of Science, 5 from Egyptian Knowledge Bank (ClinicalKey), and 32 through manual searches.

After reviewing the abstracts, 1,377 studies were excluded from the analysis, and 1,041 were considered for further fulltext screening. Finally, a total of 22 studies ${ }^{14,15,18-37}$ were included in this systematic review ${ }^{38}$.(Fig. 1)

\section{Results and study characteristics}

1) Primary outcome (pain intensity) (Fig. 2)

(1) Short follow-up period: up to one month
Table 1 reviews the summary of the included subgroup studies. Studies starting from the one week follow-up period up to one month included a total of eight studies with ten comparisons ${ }^{14,18-24}$, two of them comparing occlusal splints with medications ${ }^{18,23}$, and two comparisons of soft and hard occlusal splints with a non-occluding palatal splint ${ }^{19}$. Two studies compared occlusal splints versus exercise ${ }^{14,20}$, two studies compared them with counseling ${ }^{22,24}$, and the last two compared them with no treatment ${ }^{18,21}$.

Control groups in four comparisons reported better pain reduction compared to splint therapy ${ }^{14,19,20}$; however, the other six comparisons favored splint therapy ${ }^{18,21-24}$.

Total subgroup analysis of the eight identified studies with a total of 396 participants revealed non-significant overall effects on pain reduction (SMD $-0.11,95 \% \mathrm{CI}-0.31$ to 0.08 , $P=0.67, \mathrm{I}^{2}=0 \%$ ).

(2) Short term evaluation: up to two months

Studies with a follow-up period greater than one month and up to two months were included. Subgroups included eight studies with nine comparisons ${ }^{18-22,25-27}$. Three comparisons involved non-occluding palatal splints ${ }^{19,25,26}$, three comparisons involved exercise $\mathrm{e}^{20,21,25}$, and one study compared occlusal splints versus counselling ${ }^{22}$ with a single comparison for every medical treatment ${ }^{18}$ and cognition ${ }^{27}$.

In five comparisons, the control group exhibited better results than splints ${ }^{18-20,25,26}$, with four comparisons in favor of splints ${ }^{18,19,22,27}$. The total subgroup analysis of the identified studies with a total of 385 participants revealed non- 
Table 1. Summary of the included studies

\begin{tabular}{|c|c|c|c|c|c|c|c|c|}
\hline $\begin{array}{c}\text { Study } \\
\text { (first author) }\end{array}$ & $\begin{array}{c}\text { Publication } \\
\text { year }\end{array}$ & $\begin{array}{c}\text { Diagnostic } \\
\text { mean }\end{array}$ & Diagnosis & $\begin{array}{c}\text { Pain } \\
\text { measurement } \\
\text { tool }\end{array}$ & $\begin{array}{c}\text { Study } \\
\text { splint type }\end{array}$ & Control & $\begin{array}{c}\text { No. of } \\
\text { participants }\end{array}$ & $\begin{array}{l}\text { Follow-up } \\
\text { period }\end{array}$ \\
\hline Minakuchi $^{18}$ & 2001 & Clinical & DD & VAS & OS & NSAID & 48 & $4 \mathrm{wk}$ \\
\hline Minakuchi $^{18}$ & 2001 & Clinical & DD & VAS & OS & No treatment & 46 & $4 \mathrm{wk}$ \\
\hline Alencar $\mathrm{Jr}^{19}$ & 2009 & Clinical & MPD & SSI & Soft splint & NOS & 28 & $1 \mathrm{mo}$ \\
\hline Alencar $\mathrm{Jr}^{19}$ & 2009 & Clinical & MPD & SSI & Hard splint & NOS & 28 & $1 \mathrm{mo}$ \\
\hline Haketa $^{20}$ & 2010 & Clinical & $\mathrm{DD}$ & VAS & OS & Exercise & 44 & $4 \mathrm{wk}$ \\
\hline Niemelä $^{24}$ & 2012 & RDC/TMD & MPD & VAS & SS & Counseling & 76 & $1 \mathrm{mo}$ \\
\hline Giannakopoulos $^{21}$ & 2016 & RDC/TMD & MPD & NRS & Soft splint & No treatment & 24 & $2 \mathrm{wk}$ \\
\hline Hasanoglu Erbasar ${ }^{22}$ & 2017 & RDC/TMD & MPD & VAS & NTI-tss & Counseling & 40 & $3 \mathrm{wk}$ \\
\hline Hosgor $^{23}$ & 2017 & RDC/TMD & $\mathrm{DD}$ & VAS & SS & NSAID & 20 & $1 \mathrm{mo}$ \\
\hline Giannakopoulos $^{14}$ & 2018 & RDC/TMD & MPD & NRS & Michigan splint & Exercise & 42 & $2 \mathrm{wk}$ \\
\hline Raphael $^{26}$ & 2001 & RDC/TMD & MPD & PI & Hard splint & NOS & 63 & $6 \mathrm{wk}$ \\
\hline Carmeli $^{25}$ & 2001 & Clinical & $\mathrm{DD}$ & PI & Soft splint & Exercise & 36 & $5 \mathrm{wk}$ \\
\hline Minakuchi $^{18}$ & 2001 & Clinical & DD & VAS & OS & NSAID & 48 & $2 \mathrm{mo}$ \\
\hline Alencar $\mathrm{Jr}^{19}$ & 2009 & Clinical & MPD & SSI & Soft splint & NOS & 28 & $2 \mathrm{mo}$ \\
\hline Alencar $\mathrm{Jr}^{19}$ & 2009 & Clinical & MPD & SSI & Hard splint & NOS & 28 & $2 \mathrm{mo}$ \\
\hline Shedden Mora ${ }^{27}$ & 2013 & $\mathrm{RDC} / \mathrm{TMD}$ & $\mathrm{DD}$ & VAS & OS & Cognition & 56 & $2 \mathrm{mo}$ \\
\hline Giannakopoulos $^{21}$ & 2016 & RDC/TMD & MPD & NRS & Soft splint & No treatment & 24 & $6 \mathrm{wk}$ \\
\hline Hasanoglu Erbasar ${ }^{22}$ & 2017 & $\mathrm{RDC} / \mathrm{TMD}$ & MPD & VAS & NTI-tss & Counseling & 40 & $6 \mathrm{wk}$ \\
\hline Haketa $^{20}$ & 2010 & Clinical & $\mathrm{DD}$ & VAS & OS & Exercise & 44 & $8 \mathrm{wk}$ \\
\hline Wahlund $^{29}$ & 2003 & $\mathrm{RDC} / \mathrm{TMD}$ & MPD & PI & SS & $\mathrm{BI}$ & 81 & $3 \mathrm{mo}$ \\
\hline Wahlund $^{29}$ & 2003 & RDC/TMD & MPD & PI & SS & Relaxation & 81 & $3 \mathrm{mo}$ \\
\hline Truelove & 2006 & $\mathrm{RDC} / \mathrm{TMD}$ & MPD & PI & Hard splint & Self-care & 108 & $3 \mathrm{mo}$ \\
\hline Truelove $^{30}$ & 2006 & RDC/TMD & MPD & PI & Soft splint & Self-care & 110 & $3 \mathrm{mo}$ \\
\hline Wassell $^{28}$ & 2006 & Clinical & MPD & VAS & SS & NOS & 72 & $3 \mathrm{mo}$ \\
\hline Alencar $\mathrm{Jr}^{19}$ & 2009 & Clinical & MPD & SSI & Soft splint & NOS & 28 & $3 \mathrm{mo}$ \\
\hline Alencar $\mathrm{Jr}^{19}$ & 2009 & Clinical & MPD & SSI & Hard splint & NOS & 28 & $3 \mathrm{mo}$ \\
\hline Wahlund $^{32}$ & 2015 & $\mathrm{RDC} / \mathrm{TMD}$ & MPD & NRS & SS & Relaxation & 57 & $3 \mathrm{mo}$ \\
\hline Conti $^{31}$ & 2015 & RDC/TMD & DD & PI & ARS & Counseling & 21 & $3 \mathrm{mo}$ \\
\hline Conti $^{31}$ & 2015 & RDC/TMD & DD & PI & NTI-tss & Counseling & 21 & $3 \mathrm{mo}$ \\
\hline Hosgor $^{23}$ & 2017 & RDC/TMD & DD & VAS & SS & NSAID & 20 & $3 \mathrm{mo}$ \\
\hline Giannakopoulos $^{14}$ & 2018 & RDC/TMD & MPD & NRS & SS & Exercise & 42 & $3 \mathrm{mo}$ \\
\hline Kokkola $^{15}$ & 2018 & RDC/TMD & MPD & OHIP & SS & Counseling & 39 & $3 \mathrm{mo}$ \\
\hline Shedden Mora ${ }^{27}$ & 2013 & $\mathrm{RDC} / \mathrm{TMD}$ & $\mathrm{DD}$ & VAS & SS & Cognition & 56 & $6 \mathrm{mo}$ \\
\hline Katyayan $^{37}$ & 2014 & $\mathrm{RDC} / \mathrm{TMD}$ & MPD & VAS & SS & Counseling & 80 & $6 \mathrm{mo}$ \\
\hline Wahlund $^{32}$ & 2015 & RDC/TMD & MPD & NRS & SS & Relaxation & 22 & $6 \mathrm{mo}$ \\
\hline Hosgor $^{23}$ & 2017 & $\mathrm{RDC} / \mathrm{TMD}$ & DD & VAS & SS & NSAID & 20 & $6 \mathrm{mo}$ \\
\hline Kokkola $^{15}$ & 2018 & RDC/TMD & MPD & OHIP & SS & Counseling & 35 & $6 \mathrm{mo}$ \\
\hline Truelove $^{30}$ & 2006 & RDC/TMD & MPD & PI & Hard splint & Self-care & 113 & $1 \mathrm{yr}$ \\
\hline Truelove ${ }^{30}$ & 2006 & RDC/TMD & MPD & PI & Soft splint & Self-care & 103 & $1 \mathrm{yr}$ \\
\hline Qvintus $^{33}$ & 2015 & RDC/TMD & MPD & VAS & SS & Counseling & 46 & $1 \mathrm{yr}$ \\
\hline Wassell $^{28}$ & 2006 & Clinical & MPD & VAS & SS & No treatment & 39 & $1 \mathrm{yr}$ \\
\hline Kokkola $^{15}$ & 2018 & RDC/TMD & MPD & OHIP & SS & Counseling & 43 & $1 \mathrm{yr}$ \\
\hline Ekberg $^{34}$ & 2004 & Clinical & MPD & VAS & SS & NOS & 40 & $1 \mathrm{yr}$ \\
\hline
\end{tabular}

(DD: disk displacement, VAS: visual analogue scale, OS: occlusal splint, NSAID: nonsteroidal antiinflammatory drugs, MPD: myofascial pain dysfunction, SSI: symptom severity index, NOS: non-occluding splint, RDC/TMD: research diagnostic criteria of temporomandibular dysfunction, SS: stabilizing splint, NRS: numeric rating scale, NTI-tss: nociceptive trigeminal inhibition-tension suppression system, PI: pain intensity, BI: brief information, ARS: anterior reposition splint, OHIP: oral health impact profile)

Atef Abdel Hameed Fouda: No evidence on the effectiveness of oral splints for the management of temporomandibular joint dysfunction pain in both short and long-term follow-up systematic revie. J Korean Assoc Oral Maxillofac Surg 2020

significant overall effects on pain reduction (SMD $0.18,95 \%$ CI -0.02 to $0.38, P=0.08, \mathrm{I}^{2}=16 \%$ ).

(3) Intermediate follow-up period: three months

Nine studies with thirteen comparisons at the three months' follow-up period were included ${ }^{14,15,19,23,28-32}$. One study compared occlusal splints with medications ${ }^{23}$, two studies involved occlusal splints with a non-occluding palatal splint ${ }^{19}$, and three studies compared occlusal splints with counsel- ling $^{15,31}$. Two studies by Wahlund et al. ${ }^{29,32}$ compared splints with relaxation, and two comparisons with self-care ${ }^{30}$.

In twelve studies, the control group exhibited better results than the splints ${ }^{14,15,19,23,28-31}$, with one study in favor of splints ${ }^{32}$.

Total subgroup analysis of the identified studies with a total of 704 participants revealed a significant overall effect on pain reduction in favor of the control (SMD 0.18, 95\% CI 
0.03-0.33, $\left.P=0.02, \mathrm{I}^{2}=0 \%\right)$.

(4) Long term evaluation

(i) Six months

Five studies at the six months' follow-up period ${ }^{15,23,27,32,37}$ were included with one study comparing occlusal splints with medications $^{23}$. Two comparisons between splint and counselling $^{15,37}$ One study compared splints with relaxation ${ }^{32}$, while the remaining study compared occlusal splints versus cognition $^{27}$.

All five studies in the control group showed better results than splints regarding pain reduction.

Total subgroup analysis of the identified studies with total of 213 participants revealed a significant overall effect on pain reduction in favor of the control (SMD 0.31, 95\% CI 0.03-0.58, $P=0.03, \mathrm{I}^{2}=0 \%$ ).

(ii) One-year follow-up

Four studies with five comparisons at the one-year followup period ${ }^{15,28,30,33}$ were included. One study compared occlusal splints with no treatment ${ }^{28}$, two studies compared splints and counselling ${ }^{15,33}$, and two comparisons of Truelove et al. ${ }^{30}$

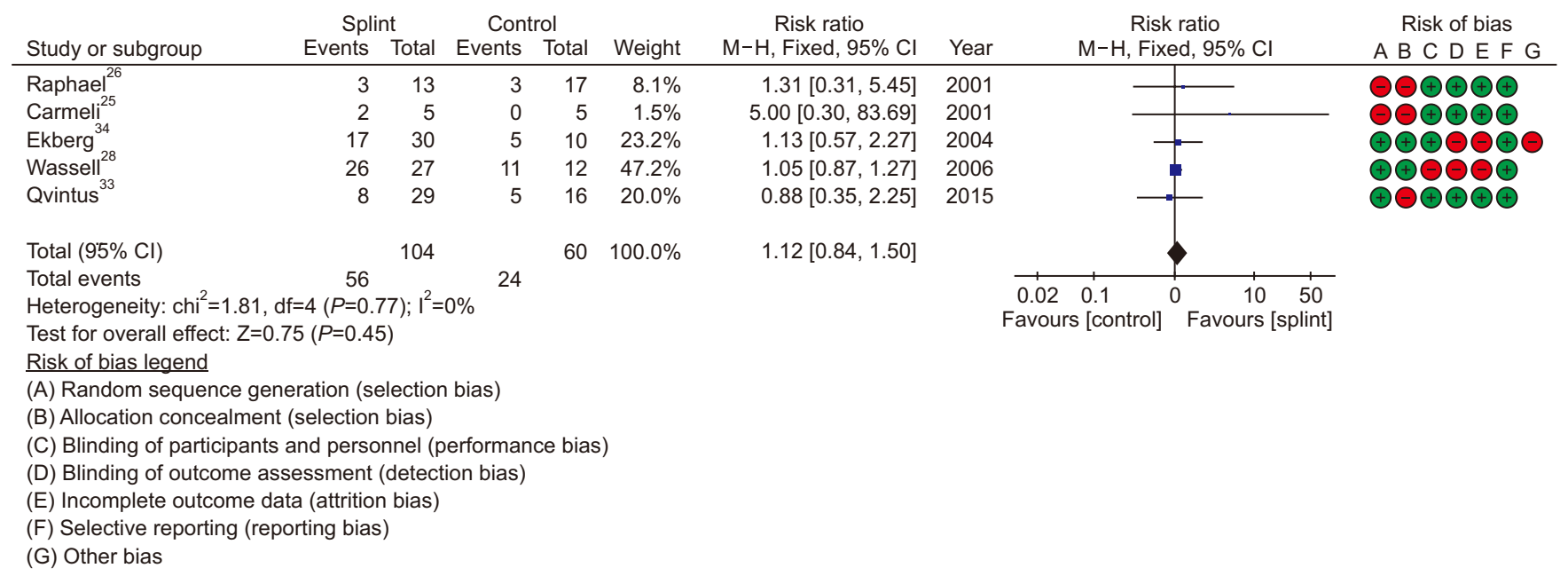

Fig. 3. Forest plot of dichotomous pain analysis at different intervals with risk of bias for the included studies. (M-H: Mantel-Haenszel test, $\mathrm{Cl}$ : confidence interval, df: degree of freedom)

Atef Abdel Hameed Fouda: No evidence on the effectiveness of oral splints for the management of temporomandibular joint dysfunction pain in both short and long-term follow-up systematic reviews and meta-analysis studies. J Korean Assoc Oral Maxillofac Surg 2020

Table 2. Summary of the included studies of short- and long-term maximum mouth opening (MMO) subgroup analysis

\begin{tabular}{|c|c|c|c|c|c|c|c|}
\hline $\begin{array}{c}\text { Study } \\
\text { (first author) }\end{array}$ & $\begin{array}{c}\text { Publication } \\
\text { year }\end{array}$ & $\begin{array}{c}\text { Diagnostic } \\
\text { mean }\end{array}$ & Diagnosis & $\begin{array}{c}\text { Pain } \\
\text { measurement } \\
\text { tool }\end{array}$ & $\begin{array}{c}\text { Study } \\
\text { splint type }\end{array}$ & Control & $\begin{array}{c}\text { No. of } \\
\text { participants }\end{array}$ \\
\hline Carmeli $^{25}$ & 2001 & Clinical & DD & MM & Soft splint & Exercise & 36 \\
\hline Minakuchi $^{18}$ & 2001 & Clinical & DD & MM & Flat OS & NSAID & 50 \\
\hline Minakuchi $^{18}$ & 2001 & Clinical & DD & MM & Flat OS & No treatment & 46 \\
\hline Niemelää & 2012 & RDC/TMD & MPD & MM & SS & Counseling & 76 \\
\hline Gomes $^{35}$ & 2014 & Clinical & MPD & MM & OS & No treatment & 28 \\
\hline Gomes $^{35}$ & 2014 & Clinical & MPD & MM & OS & Massage & 28 \\
\hline Wassell $^{28}$ & 2006 & AAOFP & MPD & MM & SS & NOS & 39 \\
\hline Wassell $^{28}$ & 2006 & AAOFP & MPD & MM & SS & NOS & 72 \\
\hline Wahlund $^{29}$ & 2003 & RDC/TMD & MPD & MM & OS & $\mathrm{BI}+$ relaxation & 83 \\
\hline Wahlund $^{29}$ & 2003 & RDC/TMD & MPD & MM & SS & $\mathrm{BI}+$ relaxation & 83 \\
\hline Wahlund $^{32}$ & 2015 & RDC/TMD & MPD & MM & SS & Relaxation & 57 \\
\hline Conti $^{31}$ & 2015 & Clinical & DD & MM & SS & Relaxation & 22 \\
\hline Conti $^{31}$ & 2015 & Clinical & DD & MM & ARS & Counseling & 21 \\
\hline Conti $^{31}$ & 2015 & Clinical & DD & MM & NTI-tss & Counseling & 21 \\
\hline
\end{tabular}

(DD: disk displacement, MM: inter-incisal opening in millimeters, OS: occlusal splint, NSAID: nonsteroidal antiinflammatory drugs, RDC/TMD: research diagnostic criteria of temporomandibular dysfunction, MPD: myofascial pain dysfunction, SS: stabilizing splint, BI: brief information, NTI-tss: nociceptive trigeminal inhibition-tension suppression system, AAOFP: American Academy of Orofacial Pain, ARS: anterior reposition splint, NOS: non-occluding splint)

Atef Abdel Hameed Fouda: No evidence on the effectiveness of oral splints for the management of temporomandibular joint dysfunction pain in both short and long-term follow-up systematic revie. J Korean Assoc Oral Maxillofac Surg 2020 
compared soft and hard splints with self-care.

In all five comparisons, the control group exhibited better results than splints regarding pain reduction.

Total subgroup analysis of the identified studies with total of 344 participants revealed a significant overall effect on pain reduction in favor of the control (SMD 0.23, 95\% CI 0.01-0.44, $P=0.04, \mathrm{I}^{2}=0 \%$ ).

\section{(5) Total analysis}

The five included subgroups reveal moderate heterogeneity between them $\left(\mathrm{I}^{2}=53.9 \%\right)$. However, no heterogeneity between the individual studies $\left(\mathrm{I}^{2}=0 \%\right)$ was observed. The overall effect of the meta-analysis with a total of 2,042 participants revealed a significant difference between the control and splint therapy in favor of the control group (SMD 0.14, $95 \%$ CI $0.05-0.23, P=0.002, \mathrm{I}^{2}=0 \%$ ).

(6) Dichotomous studies (Fig. 3)
Five studies were included ${ }^{25,26,28,33,34}$. Two studies compared manual mobilization and active exercises ${ }^{25,33}$, two studies compared splints with a palatal non-occluded splint $t^{26,34}$, and one study compared splints with no treatment ${ }^{28}$.

Total analysis of the identified studies with a total of 164 participants revealed no significant overall effects of splint therapy on pain reduction (RR 1.12, 95\% CI 0.84-1.50, $P=0.45, \mathrm{I}^{2}=0 \%$ ).

2) Secondary outcome

(1) Maximum mouth opening

(i) Short-term evaluation

Table 2 presents a summary of the included subgroup studies. Included studies involved a follow-up period up to three months. Subgroups included five studies with nine comparisons ${ }^{18,24,25,35,36}$. In five comparisons, the splint group exhibited

\begin{tabular}{|c|c|c|c|c|c|c|c|c|c|c|c|c|}
\hline \multirow[b]{2}{*}{ Study or subgroup } & \multicolumn{3}{|c|}{ Splint } & \multicolumn{3}{|c|}{ Control } & \multicolumn{3}{|c|}{ Mean difference } & \multirow{2}{*}{\multicolumn{2}{|c|}{$\begin{array}{l}\text { Mean difference } \\
\text { IV, Fixed, } 95 \% \mathrm{Cl}\end{array}$}} & \multirow{2}{*}{$\begin{array}{c}\text { Risk of bias } \\
\text { A B C D E F }\end{array}$} \\
\hline & Mean & SD & Total & Mean & SD & Total & Weight & IV, Fixed, 95\% Cl & Year & & & \\
\hline \multicolumn{13}{|l|}{ Short } \\
\hline Minakuchi $^{18}$ & 39.8 & 10.1 & 25 & 38.5 & 9.68 & 21 & $2.9 \%$ & $1.30[-4.43,7.03]$ & 2001 & & & \\
\hline Minakuchi $^{18}$ & 37.5 & 9.91 & 25 & 37 & 10.5 & 25 & $3.0 \%$ & $0.50[-5.16,6.16]$ & 2001 & & & \\
\hline Minakuchi $^{18}$ & 39.8 & 10.1 & 25 & 38.1 & 10.5 & 25 & $2.9 \%$ & $1.70[-4.01,7.41]$ & 2001 & & & \\
\hline Minakuchi $^{18}$ & 37.5 & 9.91 & 25 & 37.9 & 9.84 & 21 & $2.9 \%$ & $-0.40[-6.13,5.33]$ & 2001 & & & \\
\hline Carmeli $^{25}$ & 37.6 & 6.13 & 18 & 37.5 & 6.1 & 18 & $5.9 \%$ & $0.10[-3.90,4.10]$ & 2001 & & & \\
\hline Niemelä ${ }^{24}$ & 45.9 & 8.7 & 39 & 46.5 & 7.8 & 37 & $6.9 \%$ & $-0.60[-4.31,3.11]$ & 2012 & & & \\
\hline Gomes $^{35}$ & 47.17 & 3.53 & 14 & 49.75 & 5.35 & 14 & $8.4 \%$ & $-2.58[-5.94,0.78]$ & 2014 & & & \\
\hline Gomes $^{35}$ & 47.17 & 3.53 & 14 & 50.32 & 5.63 & 14 & $7.8 \%$ & $-3.15[-6.63,0.33]$ & 2014 & & & \\
\hline Ahmed $^{36}$ & 41.92 & 4.79 & 13 & 41.92 & 5.3 & 13 & $6.3 \%$ & $0.00[-3.88,3.88]$ & 2016 & & & \\
\hline Subtotal $(95 \% \mathrm{Cl})$ & & & 198 & & & 188 & $47.0 \%$ & $-0.87[-2.29,0.55]$ & & & & \\
\hline \multicolumn{13}{|c|}{ Heterogeneity: $\mathrm{chi}^{2}=4.66, \mathrm{df}=8(P=0.79) ; \mathrm{I}^{2}=0 \%$} \\
\hline \multicolumn{13}{|c|}{ Test for overall effect: $Z=1.20(P=0.23)$} \\
\hline \multicolumn{13}{|l|}{ Long } \\
\hline Wahlund $^{29}$ & 49.3 & 8.8 & 42 & 49.4 & 6.4 & 41 & $8.7 \%$ & $-0.10[-3.40,3.20]$ & 2003 & & & \\
\hline Wahlund $^{29}$ & 49.5 & 7.3 & 42 & 50 & 5.5 & 41 & $12.3 \%$ & $-0.50[-3.28,2.28]$ & 2003 & & & \\
\hline Wahlund $^{29}$ & 43.1 & $\begin{array}{r}1.0 \\
8\end{array}$ & $\begin{array}{l}42 \\
29\end{array}$ & 45.3 & $\begin{array}{l}5.5 \\
9.2\end{array}$ & $\begin{array}{l}41 \\
28\end{array}$ & $\begin{array}{r}12.5 \% \\
4.7 \%\end{array}$ & $-2.20[-6.68,2.28]$ & 2003 & & & \\
\hline Wassell $^{28}$ & 45.7 & 8.3 & 38 & 46.6 & 7.3 & 34 & $7.3 \%$ & $-0.90[-4.50,2.70]$ & 2006 & & & \\
\hline Wassell $^{28}$ & 47.7 & 4.1 & 27 & 48.9 & 4.1 & 12 & $12.2 \%$ & $-1.20[-3.99,1.59]$ & 2006 & & & \\
\hline Conti $^{31}$ & 41.2 & 7.2 & 12 & 39.3 & 6.1 & 9 & $2.9 \%$ & $1.90[-3.80,7.60]$ & 2015 & & & \\
\hline Conti $^{31}$ & 42.2 & 6.4 & 12 & 39.3 & 6.1 & 9 & $1.5 \%$ & $2.90[-2.48,8.28]$ & 2015 & & & \\
\hline Wahlund ${ }^{32}$ & 46.4 & $\begin{array}{l}0.4 \\
8.4\end{array}$ & 17 & 52.4 & 7.8 & 5 & $53.0 \%$ & $-6.00[-13.92,1.92]$ & 2015 & & - & \\
\hline Subtotal $(95 \% \mathrm{Cl})$ & & & 219 & & & 179 & & $-0.62[-1.96,0.72]$ & & & & \\
\hline \multicolumn{13}{|c|}{ Heterogeneity: $\mathrm{chi}^{2}=4.94, \mathrm{df}=7(P=0.67) ; \mathrm{I}^{2}=0 \%$} \\
\hline \multicolumn{13}{|c|}{ Test for overall effect: $Z=0.90(P=0.37)$} \\
\hline Total $(95 \% \mathrm{Cl})$ & & & 417 & & & 367 & $100.0 \%$ & $-0.74[-1.71,0.24]$ & & & & \\
\hline \multicolumn{12}{|c|}{ Heterogeneity: chi ${ }^{2}=9.66, \mathrm{df}=16(P=0.88) ;\left.\right|^{2}=0 \%$} & \\
\hline \multicolumn{10}{|c|}{ Test for overall effect: $Z=1.48(P=0.14)$} & $-10-5$ & $0 \quad 5 \quad 10$ & \\
\hline \multicolumn{10}{|c|}{ Test for subgroup differences: $\mathrm{chi}^{2}=0.06, \mathrm{df}=1(P=0.80) ; \mathrm{I}^{2}=0 \%$} & Favours [control] & Favours [splint] & \\
\hline \multicolumn{13}{|c|}{ Risk of bias legend } \\
\hline \multicolumn{13}{|c|}{ (A) Random sequence generation (selection bias) } \\
\hline \multicolumn{13}{|c|}{ (B) Allocation concealment (selection bias) } \\
\hline \multicolumn{13}{|c|}{ (C) Blinding of participants and personnel (performance bias) } \\
\hline \multicolumn{13}{|c|}{ (D) Blinding of outcome assessment (detection bias) } \\
\hline \multicolumn{13}{|c|}{ (E) Incomplete outcome data (attrition bias) } \\
\hline \multicolumn{13}{|c|}{ (F) Selective reporting (reporting bias) } \\
\hline (G) Other bias & & & & & & & & & & & & \\
\hline
\end{tabular}

Fig. 4. Forest plot result of comparisons of the splint group vs control group in maximum mouth opening at both short and long term durations according to the total subgroup analysis. (SD: standard deviation, Cl: confidence interval, df: degree of freedom) Atef Abdel Hameed Fouda: No evidence on the effectiveness of oral splints for the management of temporomandibular joint dysfunction pain in both short and long-term follow-up systematic reviews and meta-analysis studies. J Korean Assoc Oral Maxillofac Surg 2020 


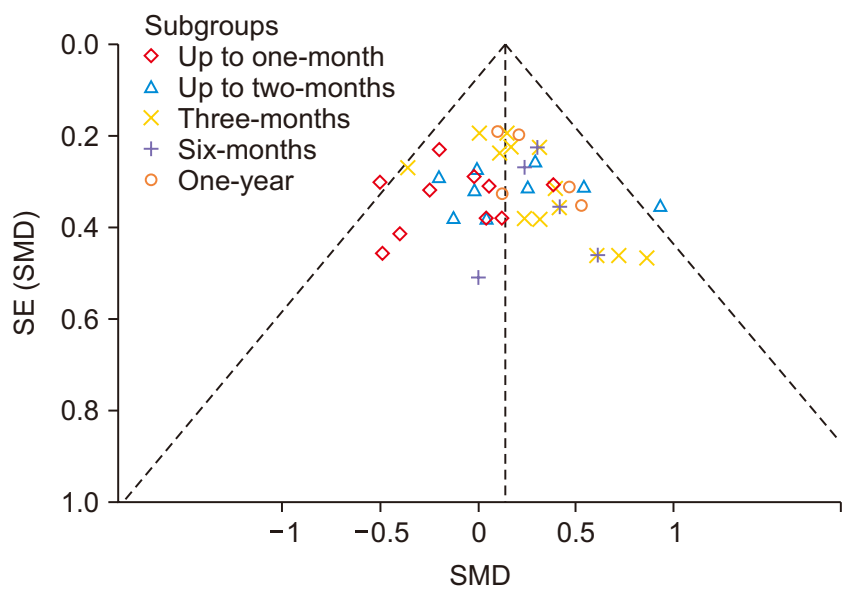

Fig. 5. Funnel plot comparison: splint group vs control group in continuous pain assessment to detect publication bias of the studies. (SE: standard error, SMD: standardized mean difference) Atef Abdel Hameed Fouda: No evidence on the effectiveness of oral splints for the management of temporomandibular joint dysfunction pain in both short and long-term follow-up systematic reviews and meta-analysis studies. J Korean Assoc Oral Maxillofac Surg 2020

better results than the non-splint group ${ }^{18,25,36}$ and four comparisons were in favor of the control ${ }^{18,24,35}$.

Total subgroup analysis of the identified studies with a total of 386 participants revealed non-significant overall effects on the improvement in mouth opening (MD $-0.87,95 \% \mathrm{CI}$ -2.29 to $0.55, P=0.23, \mathrm{I}^{2}=0 \%$ ).

(ii) Long-term evaluation

Included studies involved a follow-up period up to one year. Subgroups included four studies with eight comparisons $^{28,29,31,32}$. In two comparisons, the splint group exhibited better results than then control ${ }^{30}$, and six comparisons were in favor of the control group ${ }^{28,29,32}$.

Total subgroup analysis of the identified studies with a total of 398 participants revealed non-significant overall effects on the improvement of mouth opening (MD $-0.62,95 \% \mathrm{CI}$ -1.96 to $0.72, P=0.37 ; \mathrm{I}^{2}=0 \%$ ).

Total analysis of the identified studies with a total of 784 participants revealed no significant overall effects of splint therapy on MMO (MD $-0.74,95 \% \mathrm{CI}-1.71$ to $0.24, P=0.14$, $\mathrm{I}^{2}=0 \%$ ).(Fig. 4)

\section{3) Methodological quality assessment}

(1) Risk of bias across all the included studies

Nine studies had a high risk for selection bias (random sequence generation) (41\%), eleven studies were at high risk of selection bias (allocation concealment) (50\%), five studies had performance bias (23\%), eight studies exhibited detection bias (36\%), three studies had attrition bias (14\%), and finally two studies exhibited reporting bias (1\%).
The funnel plots declared no publication bias for the two primary outcomes, continuous and dichotomous, in both short and long-term follow-up periods (Fig. 5) and also no publication bias for the secondary outcome (MMO).

\section{Discussion}

Systematic reviews are a critical assessment tool in evidence-based decision-making. This study was assigned to PRISMA $^{16}$ statements for systematic reviews. Well-structured PICO (Problem Intervention-Comparison-Outcome) questions with clearly outlined inclusion and exclusion criteria were also included. A publication bias analysis was performed for every outcome using funnel plots.

Pain is considered the most common reason for medical consultation. Therefore, it was selected as the primary outcome of this review. However, restoring the normal range of mouth opening, and subsequently, normal masticatory and jaw function was the secondary outcome examined.

We investigated the effects of splint therapy as a conservative TMD treatment modality in reducing pain intensity and improving mouth opening through the analysis of twenty-two included studies.

There is no gold standard control to compare the different types of splints. Therefore, all the splints included in the current review were compared with no treatment or behavioral therapy.

Over-estimation of the effect of treatment resulted from an improper means of randomization, neglecting concealment of treatment allocation, not adequately blinded assessments, short follow-ups, or lacking of study power due to small sample sizes being the most serious problems.

Although several studies investigated splint therapy, the results remain controversial. Methodological differences among the studies are evident, and a comparison of the results is often difficult due to heterogeneity. The clinical relevance of these findings shows a need for more well-designed RCTs.

Studies that were older than twenty years were excluded due to the introduction of RDC/TMD classification which decreases diagnostic pitfalls and improves population selection. The use of recent materials and continuous changes in splint design all may confound the results associated with the classification's effectiveness.

In this systematic review, we tried to minimize bias across the studies and obtain maximal homogeneity among the subgroups by using appropriate eligibility criteria and selecting only studies with the control group receiving no treatment or 
palliative treatment. Subsequently, meta-regression analysis was not required.

In the current review, subgroups were selected according to the recommendation of Pficer et al. ${ }^{39}$ who studied confounding factors with meta-regression analysis and found that that duration of treatment is one of the parameters that could affect the outcome of using oral splints. He reported that investigators should pay attention not only to the short-term but also long-term therapeutic effects in their studies.

Continuous outcome data was obtained using SMD, 95\% $\mathrm{CI}$, and applying inverse variance with a fixed effect for accurate analysis. For dichotomous pain intensity data, we used relative risk and random effects because of the low number of included studies and heterogeneity.

For secondary outcomes based on continuous data, we used MD because of using the same scale in measurements and 95\% CI. Inverse variance and fixed effects were applied to achieve sensitive analysis.

In short-term follow-ups, oral splints exhibited no significant effect on pain reduction. However, in long-term followups, behavioral therapy was associated with significant pain reduction.

These findings do not mean that behavioral therapy is better than oral splints, but that the effect of splint therapy was abolished or equal to no treatment after an extended period of time.

The findings of the conducted review follow the individual studies ${ }^{18-20,24,26-28,30,33,40}$ that did not observe any difference between splint therapy and placebos.

Most of the reviews that compared splints with lasers, arthrocentesis, acupuncture, or physiotherapy resulted in fake estimations because such comparisons depend on the power of the comparator and do not provide a clue about the actual estimation.

There are several articles that included studies comparing splints with behavioral treatment, pharmacologic treatment, arthroscopy, surgical intervention, or no treatment ${ }^{41}$.

This review showed that there is no evidence to suggest that splint therapy is beneficial for pain reduction measured with different scales or even effective in reducing symptoms in patients with myofascial pain when compared with placebo or no treatment. These findings are in agreement with the systematic review by Al-Ani et al. ${ }^{42}$.

There was no evaluation of each splint type separately, and the populations were mixed including disk displacement with myofascial pain participants. Non-occluding splints were considered as controls which may have some effects through the repositioning of the tongue and patient awareness issues.

We recommend an evaluation of each type of splint separately and subgroup analysis separating cases of myofascial pain from cases of disk displacement.

\section{Conclusion}

Based on the limitations of the included studies in this systematic review due to considerable bias in selection, concealment, and blinding, the present outcome suggests that oral splints are not effective for either the reduction of pain intensity or improvement in MMO compared to control groups in patients with TMJD.

Further assessment with a higher level of evidence including studies with proper selection randomization, concealment, calculated sample size, and blinding for better estimation of the effectiveness of splint therapy in patients with TMJD are needed.

Apparent improvements observed in most of the studies on oral splints are due to the placebo effect or the natural remission of symptoms.

Oral splints can be used only as an adjunct to other non or minimally invasive treatments for TMJD management. Furthermore, oral splints could be helpful in blocking bad habits and bruxism in order to inhibit dental damage potentially induced by that disorder.

Our results confirmed that the effect of splint therapy for pain reduction in short-term durations is better than that seen in control groups, but that results becomes insignificant with fading effects in long-term studies.

\section{Author's Contributions}

The author designed the study, collect the data, and did the statistical analysis.

\section{Conflict of Interest}

No potential conflict of interest relevant to this article was reported.

\section{References}

1. Lee JY, Kim YK, Kim SG, Yun PY. Evaluation of Korean teenagers with temporomandibular joint disorders. J Korean Assoc Oral Maxillofac Surg 2013;39:231-7.

2. Chisnoiu AM, Picos AM, Popa S, Chisnoiu PD, Lascu L, Picos A, 
et al. Factors involved in the etiology of temporomandibular disorders: a literature review. Clujul Med 2015;88:473-8.

3. Garrigós-Pedrón M, La Touche R, Navarro-Desentre P, GraciaNaya M, Segura-Ortí E. Effects of a physical therapy protocol in patients with chronic migraine and temporomandibular disorders: a randomized, single-blinded, clinical trial. J Oral Facial Pain Headache 2018;32:137-50.

4. Ouanounou A, Goldberg M, Haas DA. Pharmacotherapy in temporomandibular disorders: a review. J Can Dent Assoc 2017;83:h7.

5. De la Torre Canales G, Manfredini D, Grillo CM, Guarda-Nardini L, Machado Gonçalves L, Rizzatti Barbosa CM. Therapeutic effectiveness of a combined counseling plus stabilization appliance treatment for myofascial pain of the jaw muscles: a pilot study. Cranio 2017:35:180-6.

6. Fricton J, Look JO, Wright E, Alencar FG Jr, Chen H, Lang M, et al. Systematic review and meta-analysis of randomized controlled trials evaluating intraoral orthopedic appliances for temporomandibular disorders. J Orofac Pain 2010;24:237-54.

7. Singh RK, Pal US, Goyal P, Nischal A, Gurung TR, Daga D. TMJ arthrocentesis alone and in combination with duloxetine in temporomandibular joint pain. J Maxillofac Oral Surg 2018;17:270-5.

8. Zotelli VL, Grillo CM, Gil ML, Wada RS, Sato JE, da Luz Rosário de Sousa M. Acupuncture effect on pain, mouth opening limitation and on the energy meridians in patients with temporomandibular dysfunction: a randomized controlled trial. J Acupunct Meridian Stud 2017;10:351-9.

9. Xu GZ, Jia J, Jin L, Li JH, Wang ZY, Cao DY. Low-level laser therapy for temporomandibular disorders: a systematic review with meta-analysis. Pain Res Manag 2018;2018:4230583.

10. Balaji SM, Balaji P. Surgical management of chronic temporomandibular joint dislocations. Indian J Dent Res 2018;29:455-8.

11. Klasser GD, Greene CS. Oral appliances in the management of temporomandibular disorders. Oral Surg Oral Med Oral Pathol Oral Radiol Endod 2009;107:212-23.

12. Ekberg E, Vallon D, Nilner M. The efficacy of appliance therapy in patients with temporomandibular disorders of mainly myogenous origin. A randomized, controlled, short-term trial. J Orofac Pain 2003;17:133-9.

13. Conti PC, de Alencar EN, da Mota Corrêa AS, Lauris JR, Porporatti AL, Costa YM. Behavioural changes and occlusal splints are effective in the management of masticatory myofascial pain: a short-term evaluation. J Oral Rehabil 2012;39:754-60.

14. Giannakopoulos NN, Rauer AK, Hellmann D, Hugger S, Schmitter M, Hugger A. Comparison of device-supported sensorimotor training and splint intervention for myofascial temporomandibular disorder pain patients. J Oral Rehabil 2018;45:669-76.

15. Kokkola O, Suominen AL, Qvintus V, Myllykangas R, Lahti S, Tolvanen M, et al. Efficacy of stabilisation splint treatment on the oral health-related quality of life-a randomised controlled one-year follow-up trial. J Oral Rehabil 2018;45:355-62.

16. Moher D, Liberati A, Tetzlaff J, Altman DG. Preferred reporting items for systematic reviews and meta-analyses: the PRISMA statement. Ann Intern Med 2009;151:264-9, W64.

17. Higgins JPT, Green S. Cochrane Handbook for Systematic Reviews of Interventions Version 5.1.0 [Internet]. London: The Cochrane Collaboration [cited 2019 Mar 25]. Available from: https:// handbook-5-1.cochrane.org/.

18. Minakuchi H, Kuboki T, Matsuka Y, Maekawa K, Yatani H, Yamashita A. Randomized controlled evaluation of non-surgical treatments for temporomandibular joint anterior disk displacement without reduction. J Dent Res 2001;80:924-8.

19. Alencar F Jr, Becker A. Evaluation of different occlusal splints and counselling in the management of myofascial pain dysfunction. $\mathrm{J}$ Oral Rehabil 2009;36:79-85.

20. Haketa T, Kino K, Sugisaki M, Takaoka M, Ohta T. Randomized clinical trial of treatment for TMJ disc displacement. J Dent Res 2010;89:1259-63.
21. Giannakopoulos NN, Katsikogianni EN, Hellmann D, Eberhard L, Leckel M, Schindler HJ, et al. Comparison of three different options for immediate treatment of painful temporomandibular disorders: a randomized, controlled pilot trial. Acta Odontol Scand 2016;74:480-6.

22. Hasanoglu Erbasar GN, Alpaslan C, Eroglu Inan G. Can an NTItss device be effective as a first-line therapy in patients with TMD myofascial pain? J Oral Rehabil 2017;44:589-93.

23. Hosgor H, Bas B, Celenk C. A comparison of the outcomes of four minimally invasive treatment methods for anterior disc displacement of the temporomandibular joint. Int J Oral Maxillofac Surg 2017;46:1403-10.

24. Niemelä K, Korpela M, Raustia A, Ylöstalo P, Sipilä K. Efficacy of stabilisation splint treatment on temporomandibular disorders. J Oral Rehabil 2012;39:799-804.

25. Carmeli E, Sheklow SL, Bloomenfeld I. Comparative study of repositioning splint therapy and passive manual range of motion techniques for anterior displaced temporomandibular discs with unstable excursive reduction. Physiotherapy 2001;87:26-36.

26. Raphael KG, Marbach JJ. Widespread pain and the effectiveness of oral splints in myofascial face pain. J Am Dent Assoc 2001;132:305-16.

27. Shedden Mora MC, Weber D, Neff A, Rief W. Biofeedback-based cognitive-behavioral treatment compared with occlusal splint for temporomandibular disorder: a randomized controlled trial. Clin J Pain 2013;29:1057-65.

28. Wassell RW, Adams N, Kelly PJ. The treatment of temporomandibular disorders with stabilizing splints in general dental practice: one-year follow-up. J Am Dent Assoc 2006;137:1089-98; quiz $1168-9$.

29. Wahlund K, List T, Larsson B. Treatment of temporomandibular disorders among adolescents: a comparison between occlusal appliance, relaxation training, and brief information. Acta Odontol Scand 2003;61:203-11.

30. Truelove E, Huggins KH, Mancl L, Dworkin SF. The efficacy of traditional, low-cost and nonsplint therapies for temporomandibular disorder: a randomized controlled trial. J Am Dent Assoc 2006;137:1099-107; quiz 1169.

31. Conti PC, Corrêa AS, Lauris JR, Stuginski-Barbosa J. Management of painful temporomandibular joint clicking with different intraoral devices and counseling: a controlled study. J Appl Oral Sci 2015;23:529-35.

32. Wahlund K, Nilsson IM, Larsson B. Treating temporomandibular disorders in adolescents: a randomized, controlled, sequential comparison of relaxation training and occlusal appliance therapy. J Oral Facial Pain Headache 2015;29:41-50.

33. Qvintus V, Suominen AL, Huttunen J, Raustia A, Ylöstalo P, Sipilä $\mathrm{K}$. Efficacy of stabilisation splint treatment on facial pain - 1-year follow-up. J Oral Rehabil 2015;42:439-46.

34. Ekberg E, Nilner M. Treatment outcome of appliance therapy in temporomandibular disorder patients with myofascial pain after 6 and 12 months. Acta Odontol Scand 2004;62:343-9.

35. Gomes CA, Politti F, Andrade DV, de Sousa DF, Herpich CM, Dibai-Filho AV, et al. Effects of massage therapy and occlusal splint therapy on mandibular range of motion in individuals with temporomandibular disorder: a randomized clinical trial. J Manipulative Physiol Ther 2014;37:164-9.

36. Ahmed MA, Rahman QB, Uddin MW, Asaduzzaman M, Ali MH. Efficacy of acrylic splint in management of internal derangement of temperomandibular joint. Bangladesh Med Res Counc Bull 2016;42:72-7.

37. Katyayan PA, Katyayan MK, Shah RJ, Patel G. Efficacy of appliance therapy on temporomandibular disorder related facial pain and mandibular mobility: a randomized controlled study. J Indian Prosthodont Soc 2014;14:251-61.

38. Moher D, Liberati A, Tetzlaff J, Altman DG; PRISMA Group. Preferred reporting items for systematic reviews and meta-analyses: 
the PRISMA statement. PLoS Med 2009;6:e1000097.

39. Pficer JK, Dodic S, Lazic V, Trajkovic G, Milic N, Milicic B. Occlusal stabilization splint for patients with temporomandibular disorders: meta-analysis of short and long term effects. PLoS One 2017;12:e0171296.

40. Pettengill CA, Growney MR Jr, Schoff R, Kenworthy CR. A pilot study comparing the efficacy of hard and soft stabilizing appliances in treating patients with temporomandibular disorders. J Prosthet Dent 1998;79:165-8.

41. Kim SG. Clinical treatment for symptoms associated with temporomandibular disorder. J Korean Assoc Oral Maxillofac Surg 2014:40:153-4.

42. Al-Ani MZ, Davies SJ, Gray RJ, Sloan P, Glenny AM. Stabilisation splint therapy for temporomandibular pain dysfunction syndrome. Cochrane Database Syst Rev 2004;(1):CD002778.

How to cite this article: Fouda AAH. No evidence on the effectiveness of oral splints for the management of temporomandibular joint dysfunction pain in both short and long-term followup systematic reviews and meta-analysis studies. J Korean Assoc Oral Maxillofac Surg 2020;46:87-98. https://doi.org/10.5125/ jkaoms.2020.46.2.87 